\title{
SOME BIFURCATION PROBLEMS IN CHOLESTERIC LIQUID CRYSTAL THEORY
}

\author{
by P. J. BARRATT and C. FRASER
}

(Received 26th April 1982)

\section{Introduction}

A liquid crystal is a transversely isotropic liquid consisting of large, relatively rigid, elongated molecules which align more or less parallel to their neighbours. Three distinct types of liquid crystal occur, namely nematic, cholesteric and smectic. In the absence of any external influences, nematics tend to orientate with their anisotropic axis uniformly aligned, whereas cholesterics prefer a characteristic helical configuration and smectics are more highly organised in layered structures. However, it is possible to influence the orientation of the anisotropic axis by a variety of external means. In particular, solid surfaces affect the alignment through the action of surface torques, while electromagnetic fields exert body torques which tend to align the anisotropic axis either parallel or perpendicular to the applied field. Detailed descriptions of the physical properties of liquid crystals may be found in the books by de Gennes [1] and Chandrasekhar [2] and the review by Stephen and Straley [3].

There have been a variety of experiments to investigate the competition between the orientational effects of electromagnetic fields and solid boundaries. One of the first, performed by Freedericksz and Zvolina [4], involves a sample of nematic liquid crystal at rest in a small gap between parallel plates, where suitable prior treatment of the solid surfaces leads to an initial alignment of the anisotropic axis uniformly parallel to the solid boundaries. Upon applying a magnetic field perpendicular to the plane of the plates, there is no distortion of the initial configuration until the field strength exceeds a critical value when one observes a smooth transition to a perturbed configuration in which the anisotropic axis tilts in the direction of the field. A somewhat similar effect of practical interest occurs by first rotating one plate in its own plane relative to the other. This produces an initial configuration in which the anisotropic axis is everywhere parallel to the solid boundaries, being constant in any plane parallel to the plates but varying uniformly with distance across the gap. This uniformly twisted nematic structure is employed in the display device described by Schadt and Helfrich [5]. When a sample of cholesteric liquid crystal is placed between parallel plates, the initial orientation of the anisotropic axis commonly exhibits the uniformly twisted configuration described above. For technical reasons cholesteric materials are of importance in display devices.

Here we examine possible equilibrium configurations that are relevant to the Freedericksz experiment in which a cholesteric liquid crystal is subjected to either a magnetic or electric field applied perpendicular to the plane of the plates. As is common 
in calculations for nematics, one first employs the strong anchoring condition in which the boundary alignment is prescribed. The analysis is then repeated for the case when a particular form of weak anchoring or couple stress boundary condition is adopted. We predict a variety of critical field strengths at which distortion may commence, and determine possible orientation patterns once the appropriate critical value is exceeded. The mathematical formulation of such problems result in some rather interesting examples of bifurcation phenomena in the theory of non-linear ordinary differential equations. At the same time it confirms in greater detail predictions made by Raynes [6].

\section{Basic equations}

Continuum theory introduces a unit vector $\mathbf{n}$, called the director, to represent the orientation of the anisotropic axis, and detailed accounts of this theory are presented in the reviews by Ericksen [7] and Leslie [8]. This paper seeks possible solutions relevant to the Freedericksz experiment in which a thin layer of cholesteric liquid crystal confined between parallel plates, a distance $2 l$ apart, is subjected to an electromagnetic field applied perpendicular to the plane of the plates.

Considering orientation patterns of the form

$$
n_{x}=\cos \theta(z) \cos \phi(z), \quad n_{y}=\cos \theta(z) \sin \phi(z), \quad n_{z}=\sin \theta(z)
$$

pertinent continuum equations describing the static isothermal behaviour of incompressible cholesteric liquid crystals are

$$
\left(\partial W / \partial \theta^{\prime}\right)^{\prime}-\partial W / \partial \theta=0, \quad\left(\partial W / \partial \phi^{\prime}\right)^{\prime}=0
$$

where the prime denotes differentiation with respect to $z$. Here

$$
W=W_{d}\left(\theta, \theta^{\prime}, \phi^{\prime}\right)+W_{f}(\theta)
$$

where $W_{d}$ represents the free energy per unit volume and is given by

$$
2 W_{d}=f(\theta)\left(\theta^{\prime}\right)^{2}+g(\theta)\left(\phi^{\prime}\right)^{2}-2 k_{2} \tau \cos ^{2} \theta \phi^{\prime}+k_{2} \tau^{2}
$$

with

$$
f(\theta)=k_{1} \cos ^{2} \theta+k_{3} \sin ^{2} \theta, \quad g(\theta)=\cos ^{2} \theta\left(k_{2} \cos ^{2} \theta+k_{3} \sin ^{2} \theta\right) .
$$

In equations (2.4) and (2.5) $k_{1}, k_{2}, k_{3}$ and $\tau$ are constant material parameters. $W_{f}$ is the energy per unit volume associated with the electromagnetic field and for a magnetic field

$$
2 W_{f}=-\chi_{a} H^{2} \sin ^{2} \theta-\chi_{\perp} H^{2}
$$

where $H$ is the constant magnetic field strength and $\chi_{a}$ and $\chi_{\perp}$ are constant material 
parameters. For an electric field

$$
2 W_{f}=-D^{2} /\left(\varepsilon_{\|} \sin ^{2} \theta-\varepsilon_{\perp} \cos ^{2} \theta\right),
$$

$D$ being related to the constant voltage $V$ applied across the plates through the relation

$$
V=D \int_{-l}^{l}\left(\varepsilon_{\|} \sin ^{2} \theta+\varepsilon_{\perp} \cos ^{2} \theta\right)^{-1} d z
$$

where $\varepsilon_{\|}$and $\varepsilon_{\perp}$ are constant material parameters. It is customary to assume that

$$
k_{1}>0, \quad k_{2}>0, \quad k_{3}>0 \text { and } \chi_{a}>0 .
$$

We also note that, since $\chi_{a}$ is generally small compared with $\chi_{\perp}$, one assumes that the interaction between a magnetic field and the liquid crystal is negligible and hence the field strength is constant throughout the sample. However, as Deuling [9] discusses, $\varepsilon_{a}$ defined by

$$
\varepsilon_{a}=\varepsilon_{\|}-\varepsilon_{\perp}
$$

is not small compared with $\varepsilon_{\perp}$. Hence the interaction between an electric field and the liquid crystal cannot be ignored and the field strength does not remain constant throughout the layer. For this reason the problems concerning magnetic and electric fields must be considered separately.

Employing a strong anchoring condition, where prior treatment of the bounding surfaces dictates a prescribed orientation at the plates, one seeks solutions of equations (2.2) subject to the boundary conditions

$$
\left.\begin{array}{l}
\theta(-l)=0, \quad \theta(l)=0 \quad \text { or } n \pi, \\
\phi(-l)=-\phi_{0}, \quad \phi(l)=\phi_{0} \quad \text { or } \quad \phi_{0}+m \pi,
\end{array}\right\}
$$

where $m$ and $n$ are integers. Given that a variety of solutions are possible, we follow Dafermos $[10]$ and assume that the solution most likely to occur in practice is that which minimises the energy function

$$
E=\int_{-1}^{l} W d z
$$

His experience suggests that possible solutions in which the range of $\theta$ exceeds $\pi / 2$ are associated with energies larger than those whose ranges do not. In addition, it can be shown that if $\theta(z)$ is a solution then so is $-\theta(z)$. For these reasons, we simplify the analysis throughout by assuming that $\theta \in[0, \pi / 2]$.

As an alternative to the strong anchoring condition, one might employ a weak anchoring or couple stress boundary condition at the plates. For definiteness, we adopt 
at both surfaces the relatively simple interfacial energy

$$
w=A(\boldsymbol{v} \cdot \mathbf{n})^{2}+B, \quad A>0,
$$

where $A$ and $B$ are constants and $v$ is the outward unit normal, although some generalisations are possible. With this choice, appropriate couple stress conditions are

$$
g(\theta) \phi^{\prime}=k_{2} \tau \cos ^{2} \theta, \quad f(\theta) \theta^{\prime} \pm A \sin 2 \theta=0 \quad \text { on } \quad z= \pm l
$$

We now anticipate that the solution most likely to appear is that which minimises the energy function

$$
\widetilde{E}(\bar{\theta})=\int_{-l}^{l} W d z+2 A \sin ^{2} \bar{\theta}
$$

where $\theta$ is the boundary value of $\theta$.

\section{Problem 1. Magnetic field with strong anchoring}

One obvious solution of equations (2.2) which satisfies the boundary conditions (2.11) is the uniformly twisted planar configuration

$$
\theta=0, \quad \phi=\phi_{0} z / l,
$$

and other similar possible configurations are

$$
\theta=0, \quad \phi=\left\{\left(2 \phi_{0}+m \pi\right) z+m l \pi\right\} / 2 l
$$

Of these solutions, (3.1) is associated with the least energy provided

$$
\phi_{0}-\pi / 4<l \tau<\phi_{0}+\pi / 4
$$

For definiteness, we select a fixed value for $\phi_{0}$ and given that applications employ a $\pi / 2$ twist we choose $\phi_{0}$ to be $\pi / 4$. As a consequence of (3.3), $\tau$ is taken to be positive in this paper so that configurations of the form (3.2) may be ignored in the following analysis.

Apart from the basic initial alignment (3.1), non-parallel twisted distortions in which both $\theta$ and $\phi$ vary with $z$ are also possible. As Leslie [11] discusses, equation (2.2) 2 integrates immediately to give

$$
g(\theta) \phi^{\prime}=k_{2} \tau \cos ^{2} \theta+a,
$$

while a suitable combination of $(2.2)_{1}$ and $(2.2)_{2}$ readily integrates to yield

$$
f(\theta)\left(\theta^{\prime}\right)^{2}+g(\theta)\left(\phi^{\prime}\right)^{2}+\chi_{a} H^{2} \sin ^{2} \theta=b,
$$

where $a$ and $b$ are constants of integration. Symmetry considerations suggest that one 
examines distortions in which

$$
\theta(z)=\theta(-z)
$$

with

$$
\theta^{\prime}(o)=0, \quad \theta(o)=\theta_{m}
$$

where $\theta_{m}$ is a positive parameter to be determined. Using (3.4) and (3.7) in (3.5) and then integrating, one obtains $z$ in the form

$$
z+l=\int_{0}^{\theta}\left\{f(\zeta) / F\left(\zeta, \theta_{m}\right)\right\}^{\frac{1}{2}} d \zeta, \quad-l \leqq z \leqq 0
$$

with

$$
\begin{aligned}
F\left(\zeta, \theta_{m}\right) \equiv & \chi_{a} H^{2}\left(\sin ^{2} \theta_{m}-\sin ^{2} \zeta\right)+\left(a+k_{2} \tau \cos ^{2} \theta_{m}\right)^{2} / g\left(\theta_{m}\right) \\
& -\left(a+k_{2} \tau \cos ^{2} \zeta\right)^{2} / g(\zeta)
\end{aligned}
$$

From (2.11), (3.4) and (3.6), it follows that

$$
\phi(z)=-\phi(-z), \quad \phi(o)=0
$$

and an integration of (3.4) now gives

$$
\phi+\phi_{0}=\int_{0}^{\theta}\left\{f(\zeta) / F\left(\zeta, \theta_{m}\right)\right\}^{\frac{1}{2}}\left(a+k_{2} \tau \cos ^{2} \zeta\right) / g(\zeta) d \zeta, \quad-\phi_{0} \leqq \phi \leqq 0 .
$$

Equations (3.6), (3.8), (3.10) 1 and (3.11) give the complete solution, provided $\theta_{m}$ and $a$ satisfy the relations

$$
\begin{aligned}
l & =\int_{0}^{\theta_{m}}\left\{f(\theta) / F\left(\theta, \theta_{m}\right)\right\}^{\frac{1}{2}} d \theta, \\
\phi & =\int_{0}^{\theta_{m}}\left\{f(\theta) / F\left(\theta, \theta_{m}\right)\right\}^{\frac{1}{2}}\left(a+k_{2} \tau \cos ^{2} \theta\right) / g(\theta) d \theta .
\end{aligned}
$$

With the change of variable (cf. Dafermos [10])

$$
\sin \lambda=\sin \theta / \sin \theta_{m},
$$

in (3.12), $l$ and $\phi_{0}$ are determined by the equations

$$
\begin{aligned}
l & =\int_{0}^{\pi / 2}\left\{f(\theta) / G\left(\theta, \theta_{m}\right)\right\}^{\frac{1}{2}} / \cos \theta d \lambda, \\
\phi_{0} & =\int_{0}^{\pi / 2}\left\{f(\theta) / G\left(\theta, \theta_{m}\right)\right\}^{\frac{1}{2}}\left(a+k_{2} \tau \cos ^{2} \theta\right) /(g(\theta) \cos \theta) d \lambda,
\end{aligned}
$$


where

$$
G\left(\theta, \theta_{m}\right) \equiv F\left(\theta, \theta_{m}\right) /\left(\sin ^{2} \theta_{m}-\sin ^{2} \theta\right)
$$

Taking limits as $\theta_{m}$ tends to zero in (3.14) now results in a critical magnetic field strength $H_{c}$, given by

$$
\chi_{a} H_{c}^{2} l^{2}=k_{1} \pi^{2} / 4+\left(k_{3}-2 k_{2}\right) \phi_{0}^{2}+2 k_{2} \tau l \phi_{0}
$$

At this value a smooth transition from the configuration (3.1) to that described by (3.8) and (3.11) is possible and it follows that a real $H_{c}$ exists if and only if either

$$
k_{3} \geqq 2 k_{2} \quad \text { or } \quad \phi_{0} \leqq k_{2} \tau l\left[1+\left\{1+k_{1}\left(2 k_{2}-k_{3}\right) / k_{2}^{2} l^{2} \tau^{2}\right\}^{\frac{1}{2}}\right] /\left(2 k_{2}-k_{3}\right) .
$$

A necessary condition for the non-parallel distortion to appear as $H$ exceeds $H_{c}$ is

$$
d\left(\chi_{a} H^{2}\right) /\left.d \beta\right|_{\beta=0}>0, \quad \beta \equiv \sin ^{2} \theta_{m},
$$

and differentiation of the relations (3.14) with respect to $\beta$ yields the result

$$
2 \chi_{a} l^{2}\left(d H^{2} / d \beta\right)_{\beta=0}=k_{3} \pi^{2} / 4-\left\{p \phi_{0}^{2}+2 k_{2}\left(k_{3}-2 k_{2}\right) l \tau \phi_{0}+k_{2}^{2} l^{2} \tau^{2}\right\} / k_{2},
$$

where

$$
p \equiv k_{3}^{2}-k_{2} k_{3}+k_{2}^{2}
$$

Hence it follows that (3.18) obtains if and only if

$$
\left.\begin{array}{ll}
\phi_{0}<k_{2}\left(k_{3}-2 k_{2}\right) l \tau(-1+\sqrt{1+q}) / p, & k_{3}>2 k_{2}, \\
\phi_{0}<\left\{\left(\pi^{2}-2 l^{2} \tau^{2}\right) / 6\right\}^{\frac{1}{2}}, & k_{3}=2 k_{2}, \\
\phi_{0}<k_{2}\left(2 k_{2}-k_{3}\right) l \tau(1+\sqrt{1+q}) / p, & k_{3}<2 k_{2},
\end{array}\right\}
$$

where

$$
q \equiv p\left\{k_{3} \pi^{2} / 4 k_{2} l^{2} \tau^{2}-1\right\} /\left(k_{3}-2 k_{2}\right)^{2}
$$

provided $q$ is positive. In the special case when

$$
\phi_{0}=l \tau=\pi / 4
$$

one deduces from (2.9), (3.16) and (3.19) that a real $H_{c}$ exists and (3.18) holds provided

$$
(\sqrt{17}-3) k_{3}<4 k_{2}
$$

this having some relevance to applications. 
The difference between the total energies $E_{1}$ and $E_{0}$ per unit area associated with the configurations described by (3.8) and (3.11) and (3.1), respectively, is given by

$$
\begin{aligned}
\Delta E=E_{1}-E_{0}= & \int_{0}^{\pi / 2}\left\{\chi_{a} H^{2} \beta \cos 2 \lambda+\frac{a^{2}}{g\left(\theta_{m}\right)}+\frac{2 k_{2} \tau \phi_{0}}{l}-\frac{k_{2} \phi_{0}^{2}}{l^{2}}\right. \\
& \left.+2 a k_{2} \tau\left(\frac{\cos ^{2} \theta_{m}}{g\left(\theta_{m}\right)}-\frac{\cos ^{2} \theta}{g(\theta)}\right)+k_{2}^{2} \tau^{2}\left(\frac{\cos ^{4} \theta_{m}}{g\left(\theta_{m}\right)}-\frac{2 \cos ^{4} \theta}{g(\theta)}\right)\right\} \\
& \times\left\{\frac{\beta f(\theta)}{F\left(\theta, \theta_{m}\right)}\right\}^{\frac{1}{2}} \frac{\cos \lambda}{\cos \theta} d \lambda .
\end{aligned}
$$

Differentiation of the relations (3.25) and (3.14) readily yields

$$
\left.\frac{d(\Delta E)}{d \beta}\right|_{\beta=0}=0
$$

and a further differentiation leads to the result

$$
\left.\frac{4 l d^{2}(\Delta E)}{d \beta^{2}}\right|_{\beta=0}=-k_{3} \pi^{2} / 4+\left\{p \phi_{0}^{2}+2 k_{2}\left(k_{3}-2 k_{2}\right) l \tau \phi_{0}+k_{2}^{2} l^{2} \tau^{2}\right\} / k_{2} .
$$

One immediately observes that the conditions required to ensure (3.18) holds are identical to those required to render $\Delta E$ negative for values of $\theta_{m}$ in the neighbourhood of $\theta_{m}$ equal zero. For fixed values of $l$ and $\phi_{0}$, we therefore anticipate a smooth transition between the two configurations as $H$ exceeds $H_{c}$, given by (3.16), provided

$$
k_{2} k_{3} \pi^{2}-4\left\{p \phi_{0}^{2}+2 k_{2}\left(k_{3}-2 k_{2} l \tau \phi_{0}\right)+k_{2}^{2} l^{2} \tau^{2}\right\}>0 \text {. }
$$

If $H\left(\theta_{m}\right)$, as defined by (3.14), increases monotonically with $\theta_{m}$, one expects the nonparallel distortion to persist so long as $\Delta E$ remains negative. Finally we note that $H$ tends to infinity as $\theta_{m}$ tends to $\pi / 2$.

\section{Problem 2. Electric field with strong anchoring}

Again we are concerned with finding solutions of (2.2) subject to the conditions (2.11) and one such obvious solution is the configuration (3.1). In seeking non-parallel distortions, the relevant equations are (3.4) and

$$
f(\theta)\left(\theta^{\prime}\right)^{2}+g(\theta)\left(\phi^{\prime}\right)^{2}-D^{2} /\left(\varepsilon_{||} \sin ^{2} \theta+\varepsilon_{\perp} \cos ^{2} \theta\right)=c
$$

where $c$ is a constant of integration. By an analysis parallel to that described in Section 3 , one obtains a critical value $D_{c}$ and its derivative given by

$$
\varepsilon_{a} l^{2} D_{c}^{2} / \varepsilon_{\perp}^{2}=k_{1} \pi^{2} / 4+\left(k_{3}-2 k_{2}\right) \phi_{0}^{2}+2 k_{2} l \tau \phi_{0}
$$


and

$$
\left.\left(2 \varepsilon_{a} l^{2} / \varepsilon_{\perp}^{2}\right) \frac{d D^{2}}{d \beta}\right|_{\beta=0}=3 \varepsilon_{a}^{2} l^{2} D_{c}^{2} / \varepsilon_{\perp}^{3}+k_{3} \pi^{2} / 4-\left\{p \phi_{0}^{2}+2 k_{2}\left(k_{3}-2 k_{2}\right) l \tau \phi_{0}+k_{2}^{2} l^{2} \tau^{2}\right\} / k_{2}
$$

where $\varepsilon_{a}$ is assumed to be positive. Utilising (2.8) in (4.2) and (4.3) yields a critical voltage $V_{c}$ defined by

$$
\varepsilon_{a} V_{c}^{2}=k_{1} \pi^{2}+4 \phi_{0}^{2}\left(k_{3}-2 k_{2}\right)+8 k_{2} l \tau \phi_{0}
$$

with

$$
\left.\frac{2 d}{d \beta}\left(V^{2} \varepsilon_{a}\right)\right|_{\beta=0}=\varepsilon_{a}^{2} V_{c}^{2} / \varepsilon_{\perp}+k_{3} \pi^{2}-4\left\{p \phi_{0}^{2}+2 k_{2}\left(k_{3}-2 k_{2}\right) l \tau \phi_{0}+k_{2}^{2} l^{2} \tau^{2}\right\} / k_{2}
$$

Here

$$
\Delta E=\frac{1}{2} \int_{-l}^{l}\left\{-D^{2} /\left(\varepsilon_{\perp}+\varepsilon_{a} \beta\right)+V^{2} \varepsilon_{\perp} l^{-2} / 4+Q\right\} d z
$$

where

$$
\begin{aligned}
Q= & \frac{a^{2}}{g\left(\theta_{m}\right)}+2 a k_{2} \tau\left(\frac{\cos ^{2} \theta_{m}}{g\left(\theta_{m}\right)}-\frac{\cos ^{2} \theta}{g(\theta)}\right)+k_{2}^{2} \tau^{2}\left(\frac{\cos ^{4} \theta_{m}}{g\left(\theta_{m}\right)}-\frac{2 \cos ^{4} \theta}{g(\theta)}\right) \\
& +\frac{2 k_{2} \tau \phi_{0}}{l}-\frac{k_{2} \phi_{0}^{2}}{l^{2}}
\end{aligned}
$$

and after a lengthy but straightforward calculation, one eventually obtains the results

$$
\left.\frac{d}{d \beta}(\Delta E)\right|_{\beta=0}=0,\left.\quad \frac{d^{2}}{d \beta^{2}}(\Delta E)\right|_{\beta=0}=\left.\frac{1}{8 l} \frac{d}{d \beta}\left(V^{2} \varepsilon_{a}\right)\right|_{\beta=0} .
$$

One notes that the condition for $V$ to be a monotonic increasing function of $\theta_{m}$ in the neighbourhood of $\theta_{m}$ equal zero is identical to the condition for the energy associated with the non-parallel distortion to be less than that associated with the initial configuration (3.1). Hence for given values of $l$ and $\phi_{0}$, we expect a smooth transition between the two configurations as $V$ exceeds $V_{c}$, given by (4.4), provided

$$
k_{2} k_{3} \pi^{2}-4\left\{p \phi_{0}^{2}+2 k_{2}\left(k_{3}-2 k_{2}\right) l \tau \phi_{0}+k_{2}^{2} \tau^{2} l^{2}\right\}+k_{2} \varepsilon_{a}^{2} V_{c}^{2} / \varepsilon_{\perp}>0
$$

This condition is obviously less or more restrictive than (3.28) depending on whether $\varepsilon_{\perp}$ is positive or negative. 


\section{Problem 3. Magnetic field with weak anchoring}

The uniformly twisted planar configuration

$$
\theta=0, \quad \phi^{\prime}=\tau
$$

is one obvious solution of (2.2) subject to the conditions (2.14). In addition, non-parallel distortions of the form described by (3.6), (3.7) and (3.10) are also possible. Assuming

$$
\theta( \pm l)=\bar{\theta} \quad \text { and } \quad \phi( \pm l)= \pm \bar{\phi}
$$

it can be shown that $\theta_{m}$ and $\bar{\theta}$ are related through the equation

$$
k_{2}^{2} \tau^{2}\left\{\cos ^{4} \theta_{m} / g\left(\theta_{m}\right)-\cos ^{4} \bar{\theta} / g(\bar{\theta})\right\}+\chi_{a} H^{2}\left(\sin ^{2} \theta_{m}-\sin ^{2} \bar{\theta}\right)=A^{2} \sin ^{2} 2 \bar{\theta} / f(\bar{\theta})
$$

and for small values of $\theta_{m}$

$$
\bar{\theta}^{2}=\left(\chi_{a} H^{2}-k_{3} \tau^{2}\right) \theta_{m}^{2} /\left(\chi_{a} H^{2}-k_{3} \tau^{2}+4 A^{2} / k_{1}\right)+O\left(\theta_{m}^{4}\right)
$$

Two distinct types of solution must be investigated. In one

$$
\chi_{a} H^{2}-k_{3} \tau^{2}>0 \text { and } \pi / 2 \geqq \theta_{m} \geqq \bar{\theta} \geqq 0 \text {, }
$$

while in the other

$$
k_{3} \tau^{2}-\chi_{a} H^{2}-4 A^{2} / k_{1}>0 \quad \text { and } \quad \pi / 2 \geqq \bar{\theta} \geqq \theta_{m} \geqq 0
$$

If (5.5) holds, a possible solution is again determined by (3.6), (3.8), (3.10) and (3.11) provided one sets $a$ identically equal to zero and replaces the lower limit in the integrals by $\bar{\theta}$. With the change of variable (3.13), one finds

$$
l=\int_{\bar{\lambda}}^{\pi / 2}\left\{\frac{k_{1}\left(1+m_{1} \beta \sin ^{2} \lambda\right)\left(1+m_{2} \beta \sin ^{2} \lambda\right)\left(1+m_{2} \beta\right)}{\chi_{a} H^{2}\left(1+m_{2} \beta \sin ^{2} \lambda\right)\left(1+m_{2} \beta\right)-k_{3} \tau^{2}}\right\}^{\frac{1}{2}} \frac{d \lambda}{\left(1-\beta \sin ^{2} \lambda\right)^{\frac{1}{2}}}
$$

where

$$
\sin \bar{\lambda}=\sin \bar{\theta} / \sin \theta_{m} ; m_{i}=k_{3} / k_{i}-1, \quad(i=1,2)
$$

Taking the limit as $\beta$ tends to zero in (5.7) yields a critical magnetic field strength $H_{1 c}$ defined by

$$
\chi_{a} H_{1 c}^{2}=k_{3} \tau^{2}+k_{1}\left(\pi / 2-\bar{\lambda}_{c}\right)^{2} / l^{2}, \lambda_{c}=\sin ^{-1}\left\{\left(\chi_{a} H_{1 c}^{2}-k_{3} \tau^{2}\right) /\left(4 A^{2} / k_{1}+\chi_{a} H_{1 c}^{2}-k_{3} \tau^{2}\right)\right\}^{\frac{1}{2}} .
$$


Differentiation of (5.7) with respect to $\beta$ leads to the result that (3.18) is valid if and only if

$$
\begin{aligned}
& \frac{\sin 2 \lambda_{c}}{2}\left(\frac{k_{3}}{k_{1}}-\frac{3 k_{3} \tau^{2} m_{2}}{\chi_{a} H_{1 c}^{2}-k_{3} \tau^{2}}\right)\left(\frac{\pi}{4}-\frac{\lambda_{c}}{2}\right)+\frac{\sin ^{2} 2 \lambda_{c}}{8}\left(\frac{k_{3}}{k_{1}}-\frac{k_{3} \tau^{2} m_{2}}{\chi_{a} H_{1 c}^{2}-k_{3} \tau^{2}}\right) \\
& +\frac{\left(k_{3} m_{2} \tau^{2}-4 k_{3} A^{2} / k_{1}^{2}\right)\left(\chi_{a} H_{1 c}^{2}-k_{3} \tau^{2}\right)^{2}}{\left(4 A^{2} / k_{1}+\chi_{a} H_{1 c}^{2}-k_{3} \tau^{2}\right)^{3}}-\frac{k_{3} m_{2} \tau^{2}}{\left(4 A^{2} / k_{1}+\chi_{a} H_{1 c}^{2}-k_{3} \tau^{2}\right)}>0
\end{aligned}
$$

The energy difference per unit area $\Delta \tilde{E}$ between the two solutions is now

$$
\begin{aligned}
\Delta \widetilde{E}=\tilde{E}(\bar{\theta})-\tilde{E}(0)= & \int_{\bar{\lambda}}^{\pi / 2}\left\{\chi_{a} H^{2} \beta \cos 2 \lambda+k_{2}^{2} \tau^{2}\left(\frac{\cos ^{4} \theta_{m}}{g\left(\theta_{m}\right)}-\frac{2 \cos ^{4} \theta}{g(\theta)}+k_{2}^{-1}\right)\right\} \\
& \times\left\{\frac{\beta f(\theta)}{F\left(\theta, \theta_{m}\right)}\right\}^{\frac{1}{2}} \frac{\cos \lambda d \lambda}{\cos \theta}+2 A \sin ^{2} \bar{\theta}
\end{aligned}
$$

and differentiating this equation twice with respect to $\beta$ yields the results

$$
\left.\frac{d(\Delta \tilde{E})}{d \beta}\right|_{\beta=0}=0 \quad \text { and }\left.\quad \frac{d^{2}(\Delta \tilde{E})}{d \beta^{2}}\right|_{\beta=0}=\int_{\bar{\lambda}_{c}}^{\pi / 2} \frac{d^{2} Q_{1}}{d \beta^{2}} d \lambda-\left.2\left(\frac{d Q_{1}}{d \beta} \frac{d \bar{\lambda}}{d \beta}\right)\right|_{\beta=0}+\left.2 A \frac{d^{2}\left(\sin ^{2} \bar{\theta}\right)}{d \beta^{2}}\right|_{\beta=0}
$$

where

$$
\begin{gathered}
\left\{\left[\chi_{a} H^{2}\left(1+m_{2} \beta\right)\left(1+m_{2} \beta \sin ^{2} \lambda\right)-k_{3} \tau^{2}\right] \cos 2 \lambda\right. \\
\left.+k_{3} m_{2} \tau^{2} \beta \sin ^{2} \lambda\right\}\left(1+m_{1} \beta \sin ^{2} \lambda / 2\right)\left(1+\beta \sin ^{2} \lambda / 2\right)
\end{gathered}
$$

For fixed $l$, one thus expects a smooth transition between configurations as $H$ exceeds $H_{1 c}$ provided (5.5) and (5.10) are satisfied and the right-hand side of $(5.12)_{2}$ is negative, these results agreeing with those of Leslie [11] in the limit as $A$ tends to infinity. If $H$ increases monotonically with $\theta_{m}$, the non-parallel distortion persists so long as $\Delta \tilde{E}$ remains negative and (5.3) yields the result

$$
\begin{gathered}
\lim _{\theta_{m} \rightarrow \pi / 2}\left(\cos \bar{\theta} / \cos \theta_{m}\right)=\left\{\left(\chi_{a} H_{2}^{2}-k_{2}^{2} \tau^{2} / k_{3}\right) /\left(\chi_{a} H_{2}^{2}-k_{2}^{2} \tau^{2}-4 A^{2} / k_{3}\right)\right\}^{\frac{1}{2}}, \\
H_{2}=\lim _{\theta_{m} \rightarrow \pi / 2} H\left(\theta_{m}\right),
\end{gathered}
$$

where $\bar{\theta}$ tends to $\pi / 2$ with $\theta_{m}$. Obviously a necessary condition for this to happen is

$$
k_{3} \chi_{a} H_{2}^{2}>k_{2}^{2} \tau^{2}+4 A^{2}
$$


Employing the change of variable

$$
\cosh \lambda=\cos \theta / \cos \theta_{m}
$$

in the appropriate form of $(3.12)_{1}$, one can show that $H_{2}$ is defined by

$$
l=\frac{k_{3}^{\frac{1}{2}}}{\left(\chi_{a} H_{2}^{2} k_{3}-k_{2}^{2} \tau^{2}\right)^{\frac{3}{2}}} \cosh ^{-1}\left\{\left(\chi_{a} H_{2}^{2} k_{3}-k_{2}^{2} \tau^{2}\right) /\left(\chi_{a} H_{2}^{2} k_{3}-k_{2}^{2} \tau^{2}-4 A^{2}\right)\right\}^{\frac{1}{2}}
$$

Thus the director becomes everywhere perpendicular to the plates as $H$ exceeds the finite value $\mathrm{H}_{2}$, provided the appropriate conditions are satisfied. This phenomenon is of interest, since it is clearly impossible when there is strong anchoring at the plates, as first noted in a simpler context by Nehring, Kmetz and Scheffer [12].

If (5.6) obtains, a possible non-parallel solution is

$$
z=\int_{\theta_{m}}^{\theta}\left\{f(\zeta) / F\left(\zeta, \theta_{m}\right)\right\}^{\frac{1}{2}} d \zeta, \quad 0 \leqq z \leqq l
$$

with

$$
l=\int_{\theta_{m}}^{\bar{\theta}}\left\{f(\zeta) / F\left(\zeta, \theta_{m}\right)\right\}^{\frac{1}{2}} d \zeta
$$

where $\phi$ and $\Phi$ are found by using (5.18) in (3.4) together with the conditions $(2.14)_{1}$. With the change of variable

$$
\cosh \lambda=\sin \theta / \sin \theta_{m}
$$

(5.19) becomes

$$
\begin{aligned}
l & =\int_{0}^{\bar{\lambda}_{1}} \frac{f^{\frac{1}{2}}(\theta)}{\cos \theta}\left\{-\chi_{a} H^{2}+k_{2}^{2} k_{3} \tau^{2} \cos ^{4} \theta \cos ^{4} \theta_{m} / g(\theta) g\left(\theta_{m}\right)\right\}^{-\frac{1}{2}} d \lambda, \\
\lambda_{1} & =\cosh ^{-1}\left(\sin \bar{\theta} / \sin \theta_{m}\right),
\end{aligned}
$$

and hence the critical relationship between $l$ and $H$ is found to be

$$
l=\left\{\frac{k_{1}}{k_{3} \tau^{2}-\chi_{a} H^{2}}\right\}^{\frac{1}{2}} \cosh ^{-1}\left\{\frac{\chi_{a} H^{2}-k_{3} \tau^{2}}{\chi_{a} H^{2}-k_{3} \tau^{2}+4 A^{2} / k_{1}}\right\}^{\frac{1}{2}} .
$$

However, since one can show here that

$$
\left.\frac{d}{d \beta}(\Delta \widetilde{E})\right|_{\beta=0}>0
$$

the non-parallel configuration is not expected to appear in preference to the initial alignment (5.1) and so such solutions may be ignored. 


\section{Problem 4. Electric field with weak anchoring}

The initial alignment (5.1) is obviously one solution of (2.2) subject to (2.14). In seeking non-parallel solutions of the form described above, it follows from the field equations (3.4) and (5.1) and the boundary conditions that $\bar{\theta}$ and $\theta_{m}$ must satisfy the relationship

$$
k_{2}^{2} \tau^{2}\left\{\frac{\cos ^{4} \theta_{m}}{g\left(\theta_{m}\right)}-\frac{\cos ^{4} \bar{\theta}}{g(\bar{\theta})}\right\}+\frac{D^{2} \varepsilon_{a}\left(\sin ^{2} \theta_{m}-\sin ^{2} \bar{\theta}\right)}{\left(\varepsilon_{\perp}+\varepsilon_{a} \sin ^{2} \bar{\theta}\right)\left(\varepsilon_{\perp}+\varepsilon_{a} \sin ^{2} \theta_{m}\right)}=\frac{A^{2} \sin ^{2} 2 \bar{\theta}}{f(\bar{\theta})} .
$$

Again two types of configuration must be considered. If

$$
\varepsilon_{a} D^{2} / \varepsilon_{\perp}^{2}-k_{3} \tau^{2}>0 \quad \text { and } \quad \pi / 2 \geqq \theta_{m} \geqq \bar{\theta} \geqq 0,
$$

a parallel analysis to that in Section 5 leads to a critical voltage $V_{1 c}$ given by

$$
\varepsilon_{a} V_{1 c}^{2} / 4=k_{3} \tau^{2} l^{2}+k_{1}\left(\pi / 2-\bar{\lambda}_{2 c}\right)^{2}
$$

where

$$
\bar{\lambda}_{2 c}=\sin ^{-1}\left\{\left(D_{1 c}^{2} \varepsilon_{a} / \varepsilon_{\perp}^{2}-k_{3} \tau^{2}\right) /\left(D_{1 c}^{2} \varepsilon_{a} / \varepsilon_{\perp}^{2}-k_{3} \tau^{2}+4 A^{2} / k_{1}\right)\right\}^{\frac{1}{2}}, D_{1 c}^{2}=V_{1 c}^{2} \varepsilon_{\perp}^{2} / 4 l^{2}
$$

One also finds that

$$
\begin{aligned}
& \left.\left\{\frac{4 A^{2}}{k_{1} M}+\left(\frac{\pi}{2}-\pi_{2 c}\right)\right\} \frac{d}{d \beta}\left(\frac{V^{2} \varepsilon_{a}}{4 l^{2}}\right)\right|_{\beta=0} \\
& =\frac{M \sin 2 \bar{\lambda}_{2 c}}{2}\left\{\frac{k_{3}}{k_{1}}+3\left(\frac{D_{1 c}^{2} \varepsilon_{a}^{2}}{\varepsilon_{\perp}^{3}}-k_{3} m_{2} \tau^{2}\right) /\left(\frac{D_{1 c}^{2} \varepsilon_{a}}{\varepsilon_{\perp}^{2}}-k_{3} \tau^{2}\right)\right\}\left(\frac{\pi}{4}-\frac{\bar{\lambda}_{2 c}}{2}\right) \\
& +\frac{M \sin ^{2} 2 \bar{\lambda}_{2 c}}{8}\left\{\frac{k_{3}}{k_{1}}+\left(\frac{D_{1 c}^{2} \varepsilon_{a}^{2}}{\varepsilon_{\perp}^{3}}-k_{3} m_{2} \tau^{2}\right) /\left(\frac{D_{1 c}^{2} \varepsilon_{a}}{\varepsilon_{\perp}^{2}}-k_{3} \tau^{2}\right)\right\} \\
& +\frac{1}{M^{2}}\left(k_{3} m_{2} \tau^{2}-\frac{4 A^{2} k_{3}}{k_{1}^{2}}-\frac{D_{1 c}^{2} \varepsilon_{a}^{2}}{\varepsilon_{\perp}^{3}}\right)\left(\frac{D_{1 c}^{2} \varepsilon_{a}}{\varepsilon_{\perp}^{2}}-k_{3} \tau^{2}\right) \\
& +\left(\frac{D_{1 c}^{2} \varepsilon_{a}^{2}}{\varepsilon_{\perp}^{3}}-k_{3} m_{2} \tau^{2}\right)-\left\{\frac{4 A^{2}}{k_{1} M}+\left(\frac{\pi}{2}-\bar{\lambda}_{2 c}\right)\right\} \frac{D_{1 c}^{2} \varepsilon_{a}^{2}}{\varepsilon_{\perp}^{3}}\left(1+\frac{\sin 2 \lambda_{2 c}}{\left(\pi-2 \bar{\lambda}_{2 c}\right.}\right)
\end{aligned}
$$

where

$$
M \equiv D_{1 c}^{2} \varepsilon_{a} / \varepsilon_{\perp}^{2}-k_{3} \tau^{2}+4 A^{2} / k_{1}
$$


The energy difference per unit area is now given by

$$
\Delta \widetilde{E}=\int_{\bar{\lambda}_{2}}^{\pi / 2} Q_{2} P d \lambda+2 A \sin ^{2} \bar{\theta}
$$

where

$$
Q_{2}=k_{2} \tau^{2}\left\{\frac{\cos ^{4} \theta_{m}}{g\left(\theta_{m}\right)}-\frac{2 \cos ^{4} \theta}{g(\theta)}+1\right\}-\frac{D^{2}}{\varepsilon_{\perp}+\varepsilon_{a} \beta}+\frac{V^{2} \varepsilon_{\perp}}{4 l^{2}}
$$

and

$$
P=\left\{\frac{f(\theta)\left[\left(\varepsilon_{\perp}+\varepsilon_{a} \beta\right)\left(\varepsilon_{\perp}+\varepsilon_{a} \sin ^{2} \lambda \beta\right)\left(1+m_{2} \beta\right)\left(1+m_{2} \beta \sin ^{2} \lambda\right)\right]}{\cos \theta\left[D^{2} \varepsilon_{a}\left(1+m_{2} \beta\right)\left(1+m_{2} \sin ^{2} \lambda \beta\right)-k_{3} \tau^{2}\left(\varepsilon_{\perp}+\varepsilon_{a} \beta\right)\left(\varepsilon_{\perp}+\varepsilon_{a} \sin ^{2} \lambda \beta\right)\right]}\right\}^{\frac{1}{2}}
$$

Differentiating (6.7) twice with respect to $\beta$ now yields $(5.12)_{1}$ and

$$
\begin{aligned}
\left.\frac{d^{2}(\Delta \tilde{E})}{d \beta^{2}}\right|_{\beta=0}= & \int_{\bar{\lambda}_{2 c}}^{\pi / 2}\left\{\left.\left(\frac{k_{1}}{D_{1 c}^{2} \varepsilon_{a} / \varepsilon_{\perp}^{2}-k_{3} \tau^{2}}\right)^{\frac{1}{2}} \frac{d^{2} Q_{2}}{d \beta^{2}}\right|_{\beta=0}+\left.2\left(\frac{d Q_{1}}{d \beta} \frac{d P}{d \beta}\right)\right|_{\beta=0}\right\} d \lambda \\
& -\left.\left.\left(\frac{2 k_{1}}{D_{1 c}^{2} \varepsilon_{a} / \varepsilon_{\perp}^{2}-k_{3} \tau^{2}}\right) \frac{d Q_{1}}{d \beta}\right|_{\beta=0} \frac{d \bar{\lambda}_{2}}{d \beta}\right|_{\beta=0}+\left.2 A \frac{d^{2}}{d \beta^{2}}\left(\sin ^{2} \bar{\theta}\right)\right|_{\beta=0} .
\end{aligned}
$$

We therefore expect a smooth transition to occur as $V$ exceeds $V_{1 c}$ provided the righthand sides of (6.4) and (6.10) are positive and negative, respectively. If $V$ increases monotonically with $\theta_{m}$ and $\Delta \widetilde{E}$ remains negative, one can show that the liquid crystal aligns everywhere perpendicular to the plates as $V$ exceeds $V_{2}$ defined by

$$
l=\frac{2 k_{3}^{\frac{1}{2}}}{\left(V_{2}^{2} \varepsilon_{a}-4 k_{2}^{2} \tau^{2} l^{2} / k_{3}\right)^{\frac{4}{2}}} \cosh ^{-1}\left\{\frac{\varepsilon_{a} V_{2}^{2}-4 k_{2}^{2} \tau^{2} l^{2} / k_{3}}{\varepsilon_{a} V_{2}^{2}-4 k_{2}^{2} \tau^{2} l^{2} / k_{3}-16 A^{2} l^{2} / k_{3}}\right\}^{\frac{1}{2}} .
$$

Of course a necessary condition for this to occur is

$$
\varepsilon_{a} V_{2}^{2}>\frac{4 l^{2}}{k_{3}}\left(k_{2}^{2} \tau^{2}+4 A^{2}\right)
$$

If

$$
k_{3} \tau^{2}-\varepsilon_{a} D^{2} / \varepsilon_{\perp}^{2}-4 A^{2} / k_{1}>0 \text { and } \pi / 2 \geqq \bar{\theta} \geqq \theta_{m} \geqq 0,
$$

the condition (5.23) again results and so we disregard this case.

\section{Concluding remarks}

This paper presents analyses for several bifurcation problems that are of interest in the theory of cholesteric liquid crystals. Although one must admit that only a restricted 
class of non-parallel distortions are considered, we note that results obtained by similar analyses in the theory of nematic liquid crystals are found to agree well with experimental observations. While an examination of more general types of solution is clearly desirable, such an investigation is beyond the scope of this paper.

In common with other investigations, we employ a static stability criterion to discriminate between several possible equilibrium configurations. Apart from a preliminary analysis in a simpler context by Straughan [13], no attempt has been made to solve such stability problems using the dynamic equations. While it is clearly desirable to have the dynamic results, one would equally want the above results for comparison.

Acknowledgement. Mr C. Fraser would like to thank Professor F. M. Leslie for introducing him to this subject. Both authors are grateful to Professor F. M. Leslie for his interest and encouragement during the preparation of this manuscript.

\section{REFERENCES}

1. P. G. DE Gennes, The Physics of Liquid Crystals (Clarendon Press, Oxford, 1974).

2. S. Chandrasekhar, Liquid Crystals (University Press, Cambridge, 1977).

3. M. J. Stephen and J. P. Straley, Physics of liquid crystals, Rev. Mod. Phys. 46 (1974), 617-704.

4. F. Freedericksz and V. Zvolina, The orientation of an anisotropic liquid, Trans. Faraday Soc. 29 (1933), 919-930.

5. M. Schadt and W. Helfrich, Voltage-dependent optical activity of a twisted nematic liquid crystal, Appl. Phys. Lett. 18 (1971), 127-128.

6. E. P. Raynes, Optically active additives in twisted nematic devices, Revue de Phys. Appl. 10 (1975), 117-120.

7. J. L. Ericksen, Equilibrium theory of liquid crystals, Adv. Liquid Cryst. 2 (1976), 233-298.

8. F. M. LesLIE, Theory of flow phenomena in liquid crystals, Adv. Liquid Cryst. 4 (1979), 1-81.

9. H. J. Deuling, Deformation of nematic liquid crystals in an electric field, Mol. Cryst. and Liquid Cryst. 19 (1972), 123-131.

10. C. M. Dafermos, Stability of orientation patterns of liquid crystals subject to magnetic fields, SIAM J. Appl. Maths. 16 (1968), 1305-1318.

11. F. M. LesLIE, Distortion of twisted orientation patterns in liquid crystals by magnetic fields, Mol. Cryst. and Liquid Cryst. 12 (1970), 57-72.

12. J. Nehring, A. R. Kmetz and T. J. SCheffer, Analysis of weak-boundary-coupling effects in liquid crystal displays, J. Appl. Phys. 47 (1976), 850-857.

13. B. Straughan, Stability of uniform equilibrium states of a nematic liquid crystal, $J$. de Mecanique 19 (1980), 629-638.

UNIVERSity of StrathCLYde

Dundee College of Technology 